\title{
The technique of unilateral double lobar lung transplantation in a canine model
}

Daisuke Okutani, MD
Hiroshi Date, MD
Makio Hayama, MD
Hidetoshi Inokawa, MD
Mikio Okazaki, MD
Itaru Nagahiro, MD
Yoshifumi Sano, MD
Motoi Aoe, MD
Nobuyoshi Shimizu, MD

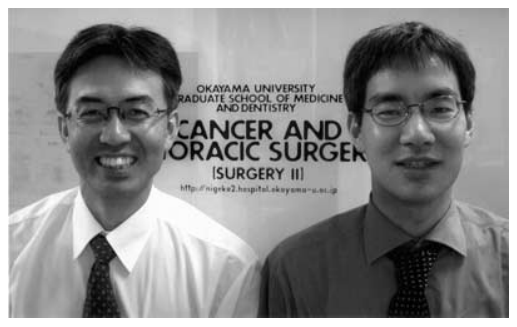

Drs Date and Okutani
From the Department of Cancer and Thoracic Surgery, Okayama University Graduate School of Medicine and Dentistry, Okayama, Japan.

This work was supported by a research grant (no. 14571266) from the Ministry of Health, Labor and Welfare, Japan.

Received for publication June 10, 2003; revisions received Aug 14, 2003; accepted for publication Aug 19, 2003.

Address for reprints: Hiroshi Date, MD, Department of Cancer and Thoracic Surgery (Surgery II), Okayama University Graduate School of Medicine and Dentistry, 2-5-1 Shikata Cho, Okayama 7008558, Japan (E-mail: hdate@nigeka2. hospital.okayama-u.ac.jp).

J Thorac Cardiovasc Surg 2004;127:563-7

0022-5223/\$30.00

Copyright (C) 2004 by The American Association for Thoracic Surgery

doi:10.1016/j.jtcvs.2003.08.045
Objective: Bilateral living-donor lobar lung transplantation has become an accepted alternative to cadaveric lung transplantation. Because only one lobe is implanted in each chest cavity, this procedure seems to be best suited for children and small adults. The purpose of this study was to develop a technique of unilateral double lobar lung transplantation that can be applied to large adult patients.

Methods: Unilateral double lobar lung transplantation was performed in 6 weightmatched pairs of dogs. In donor animals the right middle, lower, and cardiac lobes were separated as a right graft, and the left lower lobe was separated as a left graft. In recipient animals these 2 grafts were implanted in the right hemithorax after right pneumonectomy. The left graft was implanted as a right upper lobe, having been rotated $180^{\circ}$ along the vertical axis and then $180^{\circ}$ along the horizontal axis. The right graft was implanted in the natural anatomic position. Function of the transplanted grafts was assessed for 3 hours after ligation of the left main pulmonary artery while the animals were ventilated with $100 \%$ oxygen.

Results: Morphologic adaptation of the 2 grafts in the right hemithorax was found to be excellent. All 6 animals survived the assessment period with excellent pulmonary function. At the end of the 3-hour assessment period, the arterial oxygen tension was $519 \pm 31 \mathrm{~mm} \mathrm{Hg}$, and the mean pulmonary artery pressure was $30.5 \pm$ $1.7 \mathrm{~mm} \mathrm{Hg}$.

Conclusions: Unilateral double lobar lung transplantation was technically possible and associated with satisfactory early pulmonary function in a canine experimental model.

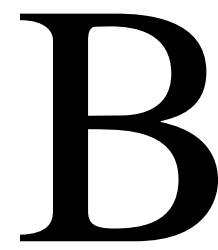

ilateral living-donor lobar lung transplantation has become an accepted approach for patients with end-stage pulmonary disease. Transplantation of lobes from 2 healthy living donors is a technique recently developed by Starnes and colleagues. ${ }^{1}$ However, this approach is confined to patients of small size because a limited amount of lung tissue is transplanted. Excessively small grafts might cause high pulmonary artery pressure, resulting in lung edema. ${ }^{2}$ A pleural space problem might increase the risk of empyema. Overexpansion of the donor lobes could contribute to obstructive physiology through early closure of the small airways. ${ }^{3}$ It is for this reason that large adult patients are thought to be poor candidates for bilateral living-donor lobar lung transplantation. 


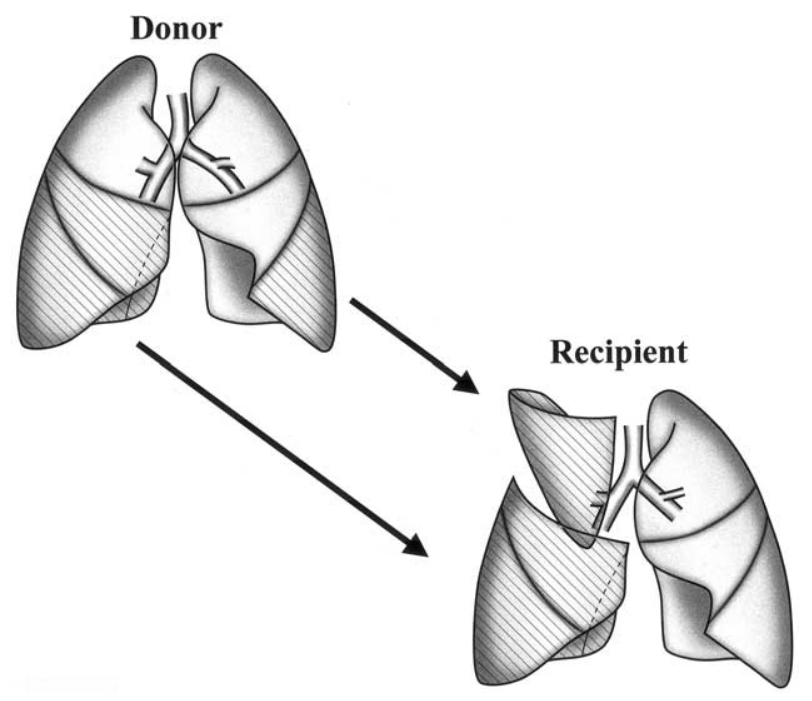

Figure 1. Unilateral double lobar lung transplantation. In the donor animal the right graft (right middle, lower, and cardiac lobes) and the left graft (left lower lobe) were separated from the double-lung block. In the recipient animal the left graft was implanted in the right upper lobe position so that the diaphragmatic aspect became the apical aspect. The right graft was implanted in the natural anatomic position.

The purpose of this experimental study was to develop a technique of unilateral double lobar lung transplantation that might be applied to large adult patients.

\section{Material and Methods}

Six weight-matched pairs of adult mongrel dogs $(19.0-27.5 \mathrm{~kg})$ were used for unilateral double lobar lung allotransplantation. The dogs were randomly selected as donors or recipients. The mean weights of donor and recipient were $23.7 \mathrm{~kg}$ and $23.5 \mathrm{~kg}$, respectively.

\section{Donor Operation}

Anesthesia was induced with intramuscular ketamine hydrochloride $(10 \mathrm{mg} / \mathrm{kg})$ and atropine sulfate $(0.01 \mathrm{mg} / \mathrm{kg})$. Intravenous thiopental sodium $(2.5 \mathrm{mg} / \mathrm{kg})$ and vecuronium bromide $(0.1 \mathrm{mg} /$ $\mathrm{kg}$ ) were used to facilitate endotracheal intubation. The donors were placed on a mechanical ventilator with a tidal volume of 20 $\mathrm{mL} / \mathrm{kg}$ and a positive end-expiratory pressure of $5 \mathrm{~cm} \mathrm{H}_{2} \mathrm{O}$ at a rate of 15 breaths/min. Anesthesia was maintained with a $0.5 \%$ inhalational halothane-oxygen mixture.

A standard median sternotomy was performed. After division of the inferior pulmonary ligaments, the pericardium was opened. The superior and inferior vena cavae were ligated and divided after systemic heparinization with sodium heparin $(200 \mathrm{U} / \mathrm{kg})$. The left atrial appendage was amputated for pulmonary venous compression. The trachea was clamped, and a double-lung block was removed without flushing and cooled in cold saline solution. The right graft (right middle, lower, and cardiac lobes) and the left graft (left lower lobe) were separated from the double-lung block at the side table (Figure 1).

\section{Recipient Operation}

Anesthesia was obtained as mentioned for the donor operation. Recipients were placed on a mechanical ventilator and maintained with a $0.5 \%$ inhalational halothane-oxygen mixture. A Swan-Ganz catheter (Baxter Healthcare Corp, Deerfield, Ill) was inserted through the femoral vein and positioned in the main pulmonary artery. Hemodynamic changes, including pulmonary artery pressure and cardiac output, were measured through this catheter by using a thermodilution method. Femoral artery cannulation with a 16-gauge catheter was used for monitoring systemic blood pressure and arterial blood gases.

A right thoracotomy was performed through the fifth intercostal space, with the animals in the left lateral position. A right pneumonectomy was performed. The first branch of the right pulmonary artery was ligated and divided. The pulmonary artery distal to this branch was divided between silk ligatures. Pulmonary veins were divided between silk ligatures placed on each venous branch at the hilum. The right upper bronchus and the truncus intermedius were divided separately. The pericardium was opened around the pulmonary veins to increase the length of recipient left atrium available for subsequent clamp placement. The endotracheal tube was advanced to the left main bronchus to facilitate the following implantation procedure.

The left graft (left lower lobe of the donor) was implanted in the right upper lobe position, and the right graft (right middle, lower, and cardiac lobes of the donor) was implanted in the natural anatomic position (Figure 1). The details of the double lobar implantation technique were as follows.

The left graft was placed in the right thorax, having been rotated $180^{\circ}$ along the vertical axis and then $180^{\circ}$ along the horizontal axis; thus in this situation, the diaphragmatic aspect of the donor left lower lobe became the apical aspect (Figure 1). The anastomosis between the donor left lower bronchus and the recipient right upper bronchus was constructed by using an end-to-end suturing technique with 5-0 Prolene sutures (Figure 2, $C$ ). Then the donor left lower pulmonary artery was anastomosed to the first branch of the recipient right pulmonary artery with 7-0 Prolene sutures. The right graft was then placed in the right chest cavity in the natural anatomic position. The anastomoses of the bronchus and the pulmonary artery were performed by using an end-to-end suturing technique. After placing a vascular clamp as proximal as possible on the recipient left atrium, a suitable cuff for the venous anastomosis was created. The 2 veins of donor grafts were approximated side by side with 7-0 Prolene sutures to form one large vein (Figure 2, A). Then it was attached to the recipient left atrial cuff (Figure 2, B). All anastomoses were easily aligned, and the implantation of both grafts did not present any difficulty (Figure 2, $C$ ). The grafts were covered with sponges and ice slush during implantation to avoid warm ischemic injury. After the vascular clamps were removed, all anastomoses were checked for air or blood leakage. A chest drain tube was inserted, and the chest was closed.

\section{Assessment of Pulmonary Function}

After transplantation, the recipient was placed in the supine position. Both lungs were ventilated with a tidal volume of $20 \mathrm{~mL} / \mathrm{kg}$, a positive end-expiratory pressure of $5 \mathrm{~cm} \mathrm{H}_{2} \mathrm{O}$, and an inspired 

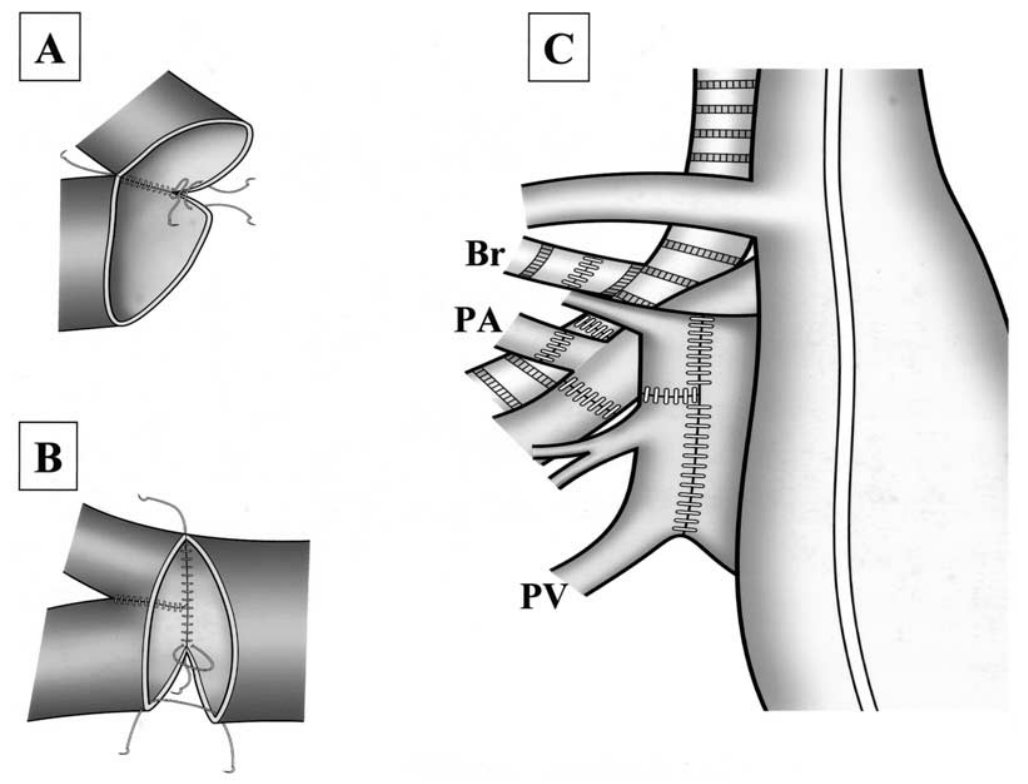

Figure 2. Alignment of the 2 donor grafts with the recipient right hilum. A, The 2 veins of the donor grafts were approximated side by side to form one large vein. B, The vein was attached to the recipient left atrial cuff. C, Completion of the 2 grafts after implantation is shown. Br, Bronchus; PA, pulmonary artery; PV, pulmonary vein.

oxygen fraction of 1.0 at a rate of 15 breaths/min. Anesthesia was maintained with an intravenous infusion of thiopental sodium. The left minithoracotomy was performed through the fifth intercostal space, and the left main pulmonary artery was ligated to allow all the cardiac output to flow to the transplanted grafts. The recipient dogs were observed for 3 hours after ligation of the left main pulmonary artery. Systemic, pulmonary, and venous pressures were continuously recorded. Measurements of cardiac output and arterial blood gas analysis were performed at specific intervals (30, 60,120 , and 180 minutes after ligation of the left pulmonary artery). Measurements were reported as the mean \pm SEM.

\section{Animal Care}

All animals received humane care in compliance with the "Principles of Laboratory Animal Care" formulated by the National Society for Medical Research and the "Guide for the Care and Use of Laboratory Animals" prepared by the Institute of Laboratory Animal Resources, National Research Council, and published by the National Academy Press, revised 1996.

\section{Results}

All 6 animals survived the operation and the 3-hour assessment period on a ventilator. The left graft implanted in the upside-down position filled the apical area of the right thoracic cavity, and there was no sign of lung congestion (Figure 3). The total ischemic time was $209 \pm 18$ minutes, and the graft implantation time was $127 \pm 7$ minutes.

The results of gas exchange and the hemodynamics of the recipients during the 3-hour assessment period are shown in Table $1 . \mathrm{PaO}_{2}$, arterial carbon dioxide tension, mean arterial pressure, mean pulmonary artery pressure, mean central venous pressure, and cardiac output were all stable during the assessment period. All 6 animals showed excellent $\mathrm{PaO}_{2}$ values throughout the observation period (Figure 4). At the end of the 3-hour assessment period, $\mathrm{PaO}_{2}$ was $519 \pm 31 \mathrm{~mm} \mathrm{Hg}$, and the mean pulmonary artery pressure was $30.5 \pm 1.7 \mathrm{~mm} \mathrm{Hg}$.

\section{Discussion}

Pulmonary transplantation from cadaveric donors is limited by the scarcity of organ availability. Bilateral living-donor lobar lung transplantation was developed by Starnes and colleagues ${ }^{1}$ as an alternative to cadaveric lung transplantation to circumvent this obstacle. In this procedure 2 healthy donors are selected. One donor is selected for removal of the right lower lobe and the other for removal of the left lower lobe. These 2 lobes are implanted in the recipient as the whole right and left lungs. Because a limited amount of lung tissue is implanted, bilateral living-donor lobar lung transplantation seems to be confined to patients of small size. We have shown in a canine animal experiment model that excessively small grafts caused high pulmonary artery pressure, resulting in lung edema. ${ }^{2}$ In a recent clinical report overexpansion of the donor lobes contributed to obstructive physiology through early closure of the small airways. ${ }^{3}$ In our clinical experience ${ }^{4}$ of bilateral living-donor lobar lung transplantation in 17 adult patients, 16 of them were women. The only male patient was relatively small, $163 \mathrm{~cm}$ in height. We have turned down a number of patients because we thought that the recipient was too big for the 

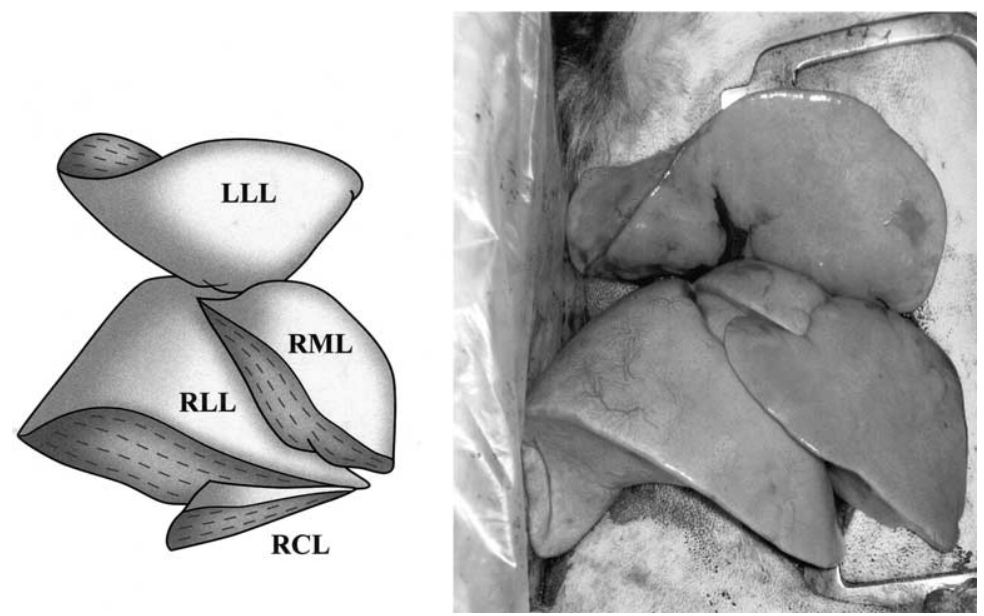

Figure 3. Macroscopic findings after double lobar lung transplantation. $L L$, Left lower lobe; $R M L$, right middle lobe; $R L L$, right lower lobe; $R C L$, right cardiac lobe. Note that the diaphragmatic aspect of the left lower lobe became the apical aspect.

TABLE 1. Assessment of lung and cardiac function of the recipient

\begin{tabular}{lcccc}
\hline & \multicolumn{4}{c}{ After transplantation } \\
\cline { 2 - 5 } & $\mathbf{3 0}$ min & $\mathbf{6 0}$ min & $\mathbf{1 2 0}$ min & $\mathbf{1 8 0}$ min \\
\hline $\mathrm{PaO}_{2}(\mathrm{~mm} \mathrm{Hg})$ & $518 \pm 30$ & $516 \pm 27$ & $526 \pm 30$ & $519 \pm 31$ \\
$\mathrm{PaCO}_{2}(\mathrm{~mm} \mathrm{Hg})$ & $34.6 \pm 4.0$ & $35.3 \pm 3.0$ & $35.1 \pm 3.3$ & $35.1 \pm 3.4$ \\
$\mathrm{Mean} \mathrm{AoP}(\mathrm{mm} \mathrm{Hg})^{132 \pm 5}$ & $130 \pm 4$ & $130 \pm 4$ & $125 \pm 7$ \\
Mean PAP (mm Hg) & $33.7 \pm 1.5$ & $33.2 \pm 2.2$ & $31.3 \pm 2.2$ & $30.5 \pm 1.7$ \\
Mean CVP (mm Hg) & $5.8 \pm 0.2$ & $6.7 \pm 0.3$ & $6.2 \pm 0.3$ & $6.0 \pm 0.4$ \\
$\mathrm{CO}(\mathrm{L} / \mathrm{min})$ & $1.2 \pm 0.1$ & $1.4 \pm 0.1$ & $1.4 \pm 0.1$ & $1.3 \pm 0.1$ \\
\hline
\end{tabular}

$\overline{\text { All values are presented as means } \pm \text { SEM. Data after transplantation were }}$ obtained with the left pulmonary artery ligated.

$\mathrm{PaO}_{2}$, Arterial oxygen tension; $\mathrm{PaCO}_{2}$; arterial carbon dioxide tension; $\mathrm{AoP}$, aortic pressure; $P A P$, pulmonary arterial pressure; $C V P$, central venous pressure; $C O$, cardiac output.

respective donors. With the hope of expanding the possibilities for transplantation in large adult patients, the present experimental study was performed.

Unilateral double lobar lung transplantation, implanting 2 grafts in the right hemithorax, is based on the notion that single-lung transplantation has been successfully performed for various noninfectious lung diseases. The remaining native lung, although it is diseased, provides some gas exchange capacity and vascular bed.

We used a canine model to develop a technique of unilateral double lobar lung transplantation. A single donor was used in this experiment to simplify the model. The right graft (right middle, lower, and cardiac lobes) resembles the human right lower lobe anatomically. The left graft (left lower lobe) resembles the human left lower lobe. Given the fact that the model has some difference from the human setting, such as chest shape and vascular anatomy, we

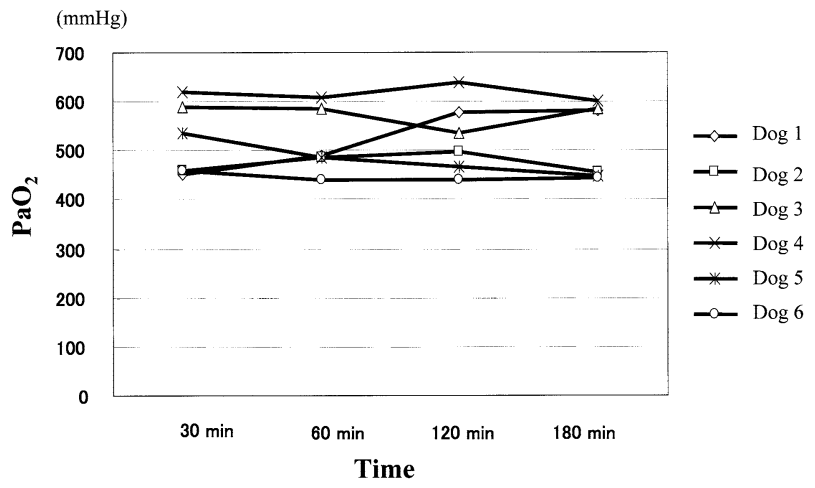

Figure 4. Arterial oxygen tension on a ventilator with an inspired oxygen fraction of 1.0 after ligation of the left main pulmonary artery.

believe that this model is good enough to develop a technique of unilateral double lobar lung transplantation.

Several technical points required elaboration. In the recipient it was essential that sufficient length of vascular and bronchial pedicles was left to facilitate subsequent anastomoses. Care had to be taken in positioning the left graft (left lower lobe) in the right chest cavity before anastomoses so that there was no undue tension when ventilation was restored. The left graft was positioned so that the diaphragmatic aspect became the apical aspect. The anastomosis between the left graft pulmonary artery and the first branch of the recipient right pulmonary artery was the most difficult part of this operation. A Satinsky clamp was placed as proximal as possible on the recipient right pulmonary artery. The first branch of the recipient right pulmonary artery was divided close to its take-off point to minimize the size disparity. In contrast to the left graft implantation in its 
nonanatomic position, implanting the right graft in its anatomic position was straightforward. The atrial anastomosis was performed last. Avoiding 2 separate venous anastomoses, the 2 veins were approximated side by side to form one large vein.

Implanting a lobe in its nonanatomic position has been reported by Couetil and associates. ${ }^{5}$ They split the donor left lung and then implanted the left lower lobe in the left hemithorax and the left upper lobe in the right hemithorax. They successfully used this split-lung transplant technique for pediatric and small adult patients. They also demonstrated normal bronchial healing after performing the anastomosis between the left upper bronchus to the intermedius bronchus.

With care of several technical points mentioned above in our experimental study, all anastomoses were easily aligned, and the implantation of both grafts did not present any difficulty. Morphologic adaptation of the 2 grafts in the right hemithorax was found to be excellent. The left graft implanted in the upside-down position filled the apical area of the right thoracic cavity. The satisfactory early pulmonary function of the transplanted grafts was confirmed by ligating the left pulmonary artery.
The quality of bronchial healing and pulmonary function on spontaneous breathing are to be evaluated. Further longer-term animal studies are required to assess the full potential of this new transplantation technique before clinical application in large adult patients.

We thank Tetsuo Kawakami for his expert technical assistance.

\section{References}

1. Starnes VA, Barr ML, Cohen RG, Hagen JA, Wells WJ, Horn MV, et al. Living-donor lobar lung transplantation experience. Intermediate results. J Thorac Cardiovasc Surg. 1996;112:1284-91.

2. Fujita T, Date H, Ueda K, Nagahiro I, Aoe M, Andou A, et al. Experimental study on size matching in a canine living-donor lobar lung transplant model. J Thorac Cardiovasc Surg. 2002;123:104-9.

3. Haddy SM, Bremner RM, Moore-Jefferies EW, Thangathurai D, Schenkel FA, Barr ML, et al. Hyperinflation resulting in hemodynamic collapse following living donor lobar transplantation. Anesthesiology. 2002;97:1315-7.

4. Date H, Aoe M, Nagahiro I, Sano Y, Andou A, Matsubara H, et al. Living-donor lobar lung transplantation for various lung diseases. J Thorac Cardiovasc Surg. 2003;126:476-81.

5. Couetil JPA, Tolan MJ, Loulmet DF, Guinvarch A, Chevalier PG, Achkar A, et al. Pulmonary bipartitioning and lobar transplantation. A new approach to donor organ shortage. J Thorac Cardiovasc Surg. 1997;113:529-37. 